\title{
A remarkable set of Schönflies-singular planar Stewart Gough platforms
}

\author{
Georg Nawratil \\ Institute of Discrete Mathematics and Geometry, Vienna University of \\ Technology, Wiedner Hauptstrasse 8-10/104, Vienna, A-1040, Austria
}

\begin{abstract}
We present a set of planar parallel manipulators of Stewart Gough type which are singular with respect to the Schönflies group $X(a)$ without being architecturally singular. This set of so called Schönflies-singular planar parallel manipulators is characterized by the property that the carrier plane of the platform or of the base anchor points is orthogonal to the rotational axis a of the Schönflies group X(a). By giving the necessary and sufficient conditions we provide a complete classification of this set. Beside this algebraic characterization we also present a geometric one. Moreover we discuss the self-motional behavior of these manipulators and prove that they possess a quadratic singularity surface.
\end{abstract}

Key words: Schönflies motion group, Stewart Gough Platform, planar parallel manipulator, Schönflies-singular manipulators, self-motions

\section{Introduction}

In this article we discuss a class of planar parallel manipulators of Stewart Gough type which are singular with respect to the Schönflies motion group $\mathrm{X}(\mathrm{a})$. This 4-dimensional group, which is named after the German geometer Arthur Moritz Schönflies (cf. [20,21]), is the largest subgroup of the Special Euclidean motion group SE(3) and includes three linearly independent translations and all rotations about a fixed axis a.

A planar parallel manipulator of Stewart Gough type consists of two systems, namely the platform $\Sigma$ and the base $\Sigma_{0}$, which are connected via six SphericalPrismatic-Spherical (or Spherical-Prismatic-Universal) joints. The geometry

Email address: nawratil@geometrie.tuwien.ac.at (Georg Nawratil).

URL: http://www.geometrie.tuwien.ac.at/nawratil (Georg Nawratil). 
of such a manipulator is given by the six base anchor points $\mathrm{M}_{i} \in \Sigma_{0}$ with coordinates $\mathbf{M}_{i}:=\left(A_{i}, B_{i}, 0\right)^{T}$ and by the six platform anchor points $\mathbf{m}_{i} \in \Sigma$ with coordinates $\mathbf{m}_{i}:=\left(a_{i}, 0, b_{i}\right)^{T}$. By using Euler parameters $\left(e_{0}, e_{1}, e_{2}, e_{3}\right)$ for the parametrization of the spherical motion group $\mathrm{SO}(3)$ the coordinates $\mathbf{m}_{i}^{\prime}$ of $\mathbf{m}_{i}$ with respect to the fixed space can be written as $\mathbf{m}_{i}^{\prime}=K^{-1} \mathbf{R} \cdot \mathbf{m}_{i}+\mathbf{t}$ with

$$
\mathbf{R}:=\left(r_{i j}\right)=\left(\begin{array}{ccc}
e_{0}^{2}+e_{1}^{2}-e_{2}^{2}-e_{3}^{2} & 2\left(e_{1} e_{2}-e_{0} e_{3}\right) & 2\left(e_{1} e_{3}+e_{0} e_{2}\right) \\
2\left(e_{1} e_{2}+e_{0} e_{3}\right) & e_{0}^{2}-e_{1}^{2}+e_{2}^{2}-e_{3}^{2} & 2\left(e_{2} e_{3}-e_{0} e_{1}\right) \\
2\left(e_{1} e_{3}-e_{0} e_{2}\right) & 2\left(e_{2} e_{3}+e_{0} e_{1}\right) & e_{0}^{2}-e_{1}^{2}-e_{2}^{2}+e_{3}^{2}
\end{array}\right)
$$

the translation vector $\mathbf{t}:=\left(t_{1}, t_{2}, t_{3}\right)^{T}$ and $K:=e_{0}^{2}+e_{1}^{2}+e_{2}^{2}+e_{3}^{2}$.

It is well known (see e.g. [10]) that a Stewart Gough platform is in a singular position if and only if the carrier lines of the prismatic legs belong to a linear line complex $\mathcal{C}$, or analytically seen, if $Q:=\operatorname{det}(\mathbf{Q})=0$ holds, where the $i^{\text {th }}$ row of the $6 \times 6$ matrix $\mathbf{Q}$ equals the Plücker coordinates $\underline{\mathbf{l}}_{i}:=\left(\mathbf{l}_{i}, \widehat{\mathbf{l}}_{i}\right):=$ $\left(\mathbf{m}_{i}^{\prime}-\mathbf{M}_{i}, \mathbf{M}_{i} \times \mathbf{l}_{i}\right)$ of the $i^{t h}$ carrier line.

\subsection{Preliminary considerations and overview}

Definition 1 A Stewart Gough platform is called Schönflies-singular (or more precisely $\mathrm{X}(\mathrm{a})$-singular) if there exists a Schönflies group $\mathrm{X}(\mathrm{a})$ such that the manipulator is singular for all transformations from $\mathrm{X}(\mathrm{a})$ (applied to the moving part of the $S G$ platform).

A special class of Schönflies-singular manipulators are the architecturally singular ones (cf. Ma and Angeles [9]) because they are singular with respect to any Schönflies group. As architecturally singular manipulators are already classified (cf. Karger [4,6], Mick and Röschel[11,19], Nawratil [14,15]) and Wohlhart [23]) we are only interested in Schönflies-singular manipulators which are not architecturally singular.

For the determination of $\mathrm{X}(\mathrm{a})$-singular planar parallel manipulators we distinguish the following cases depending on the angles between the axis a and the carrier plane $\Phi$ of the base anchor points and the carrier plane $\varphi$ of the platform anchor points, respectively. By setting $\alpha:=\angle(\mathrm{a}, \Phi) \in[0, \pi / 2]$ and $\beta:=\angle(\mathrm{a}, \varphi) \in[0, \pi / 2]$ the classification can be done as follows:

(1) $\alpha \neq \beta$
(a) $\alpha=\pi / 2$ and $\beta \in[0, \pi / 2[$
(b) $\alpha \in[0, \pi / 2[$ and $\beta \in[0, \pi / 2[$

(2) $\alpha=\beta$
(a) $\alpha=\pi / 2$
(b) $\alpha \in] 0, \pi / 2[$
(c) $\alpha=0$ 
Every Schönflies-singular manipulator belongs to one of these cases (after exchanging the platform and the base).

In this paper we give a complete classification of case (1a). The presented solution set is remarkable because it turns out (cf. [16]) that these are the only Schönflies-singular planar manipulators with $\alpha \neq \beta$ aside from the architecturally singular ones. Moreover it should be noted, that the special cases (i.e. $\alpha=\beta$ ) of Schönflies-singular planar Stewart Gough platforms are given in [17]. Therefore we also close the discussion of Schönflies-singular planar parallel manipulators which was started by Wohlhart [22] by giving an example (polygon platform) for a $\mathbf{X}(\mathrm{a})$-singular planar SG platform of case (2a).

As $\alpha \neq \beta$ holds there always exist a common line $\mathrm{s}$ of $\varphi$ and $\Phi$ and therefore we can apply the following lemma given by Mick and Röschel [11]:

Lemma 1 If the connecting lines of $\mathrm{M}_{i} \in \Phi$ and $\mathrm{m}_{i} \in \varphi$ of two intersecting planes $\Phi$ and $\varphi$ belong to a linear line complex, then this property remains unchanged under rotations of the planes about their intersection line.

As $\Phi$ is orthogonal to the axis a the rotations about a and the line s commute. Therefore the solution set of case (1a) does not depend on $\beta$ and we can assume $\beta=0$. This is the reason for choosing $\varphi$ as the $x z$-plane of $\Sigma$, i.e. $\mathbf{m}_{i}=\left(a_{i}, 0, b_{i}\right)^{T}$ while a equals the $z$-axis (i.e. $e_{1}=e_{2}=0$ ). As a consequence the solution of our problem corresponds to those non-architecturally singular manipulators which cause $Q=0$ for all values of $t_{1}, t_{2}, t_{3}, e_{0}, e_{3} .{ }^{1}$ The discussion of the resulting set of equations is split up into the following parts: The case study is started in Sec. 2, where we assume that there do not exist 4 collinear anchor points. In Sec. 3 resp. Sec. 4 we assume that at least 4 base anchor points resp. platform anchor points are collinear. In Sec. 5 we give a geometric characterization of the computed set and in Sec. 6 we discuss its self-motional behavior. We close the article by giving a final example.

\subsection{Notation}

Beside the term Schönflies-singular, we also introduce a new notation in the study on singularities of parallel manipulators. We denote the determinant of certain $j \times j$ matrices as follows:

$$
|\mathbf{X}, \mathbf{y}, \ldots, \mathbf{X y}|_{\left(i_{1}, i_{2}, \ldots, i_{j}\right)}:=\operatorname{det}\left(\mathbf{X}_{\left(i_{1}, i_{2}, \ldots, i_{j}\right)}, \mathbf{y}_{\left(i_{1}, i_{2}, \ldots, i_{j}\right)}, \ldots, \mathbf{X y}_{\left(i_{1}, i_{2}, \ldots, i_{j}\right)}\right)
$$

\footnotetext{
1 Nevertheless the Schönflies group has 4 parameters we have five unknowns $t_{1}, t_{2}, t_{3}, e_{0}, e_{3}$ as the rotational part is homogenized.
} 
with $\mathbf{X}_{\left(i_{1}, i_{2}, \ldots, i_{j}\right)}=\left[\begin{array}{c}X_{i_{1}} \\ X_{i_{2}} \\ \vdots \\ X_{i_{j}}\end{array}\right], \mathbf{y}_{\left(i_{1}, i_{2}, \ldots, i_{j}\right)}=\left[\begin{array}{c}y_{i_{1}} \\ y_{i_{2}} \\ \vdots \\ y_{i_{j}}\end{array}\right], \mathbf{X y}_{\left(i_{1}, i_{2}, \ldots, i_{j}\right)}=\left[\begin{array}{c}X_{i_{1}} y_{i_{1}} \\ X_{i_{2}} y_{i_{2}} \\ \vdots \\ X_{i_{j}} y_{i_{j}}\end{array}\right]$

and $\left(i_{1}, i_{2}, \ldots, i_{j}\right) \in\{1, \ldots, 6\}$ with $i_{1}<i_{2}<\ldots<i_{j}$. Moreover it should be noted that we write $|\mathbf{X}, \mathbf{y}, \ldots, \mathbf{X y}|_{i_{1}}^{i_{j}}$ if $i_{k+1}=i_{k}+1$ for $k=1, \ldots, j-1$ hold. This notation helps considerably proving the given theorems and its clarity allows a deeper insight into the geometry of the manipulator.

Moreover $c_{(i, j, k)}:=|\mathbf{1}, \mathbf{a}, \mathbf{b}|_{(i, j, k)}=0$ and $C_{(i, j, k)}:=|\mathbf{1}, \mathbf{A}, \mathbf{B}|_{(i, j, k)}=0$ express the collinearity of the points $\mathrm{m}_{i}, \mathrm{~m}_{j}, \mathrm{~m}_{k}$ and $\mathrm{M}_{i}, \mathrm{M}_{j}, \mathrm{M}_{k}$, respectively.

It should also be said that in the later done case study we always factor out the homogenizing factor $K$ if possible. Moreover we give the number $n$ of terms of a not explicitly given polynomial $F$ in square brackets, i.e. $F[n]$. The notation $F_{i j k}^{u v}$ denotes the coefficient of $t_{1}^{i} t_{2}^{j} t_{3}^{k} e_{0}^{u} e_{3}^{v}$ of $F[n]$. Moreover we only consider such coefficients where $u+v$ equals the degree of $F$ with respect to $e_{0}$ and $e_{3}$ (after factoring out the homogenizing factor $K$ as often as possible).

\section{No four anchor points are collinear}

The following lemma is a modified version of the one presented by Karger [4]:

Lemma 2 For any planar parallel manipulator with no four anchor points collinear, we can choose Cartesian coordinate systems in $\Sigma$ and $\Sigma_{0}$ in such a way that $A_{1}=B_{1}=B_{2}=a_{1}=b_{1}=0$ and $|\mathbf{A}, \mathbf{B}, \mathbf{B a}, \mathbf{B b}|_{2}^{5} \neq 0$ hold. Moreover we can assume $|\mathbf{A}, \mathbf{B}, \mathbf{B a}|_{2}^{4} \neq 0$ and $|\mathbf{A}, \mathbf{B}, \mathbf{B b}|_{2}^{4} \neq 0$.

Proof: For the proof of $|\mathbf{A}, \mathbf{B}, \mathbf{B a}, \mathbf{B b}|_{2}^{5}=A_{2} B_{3} B_{4} B_{5} c_{(3,4,5)} \neq 0$ we refer to [4]. As the points $\mathrm{m}_{3}, \mathrm{~m}_{4}, \mathrm{~m}_{5}$ are not collinear we can relabel them such that $\left(b_{3}-b_{4}\right)\left(a_{3}-a_{4}\right) \neq 0$. This already yields the proof.

Beside this lemma we also need the following abbreviations for the formulation of Theorem 1:

$$
\begin{array}{ll}
K_{1}:=|\mathbf{A}, \mathbf{B}, \mathbf{B a}, \mathbf{B b}, \mathbf{a}|_{2}^{6}, & L_{1}:=|\mathbf{A}, \mathbf{B}, \mathbf{B b}, \mathbf{a}|_{2}^{5}, \\
K_{2}:=|\mathbf{A}, \mathbf{B}, \mathbf{B a}, \mathbf{B b}, \mathbf{b}|_{2}^{6}, & L_{2}:=|\mathbf{A}, \mathbf{B}, \mathbf{B b}, \mathbf{b}|_{2}^{5}, \\
K_{3}:=|\mathbf{A}, \mathbf{B}, \mathbf{B a}, \mathbf{B b}, \mathbf{A a}|_{2}^{6}, & L_{3}:=|\mathbf{A}, \mathbf{B}, \mathbf{B b}, \mathbf{A b}|_{2}^{5}, \\
K_{4}:=|\mathbf{A}, \mathbf{B}, \mathbf{B a}, \mathbf{B b}, \mathbf{A b}|_{2}^{6} . &
\end{array}
$$

Theorem 1 For a planar Stewart Gough platform with no 4 collinear base anchor points we can assume Cartesian coordinate systems such that Lemma 
2 hold. Then a non-architecturally singular manipulator, where a is orthogonal to $\Phi$ and parallel to $\varphi$, is X(a)-singular in one of the following cases (after permutation of indices): Compute $A_{6}$ from $K_{1}=0$ and $A_{5}$ from $L_{1}=0$.

1. Compute $a_{6}$ from $K_{2}=0$ and $a_{5}$ from $L_{2}=0$. Moreover we compute $A_{4}$ from $L_{3}=0$ and one equation remains:

$$
\begin{array}{r}
b_{3}^{2} b_{4}^{2}\left(B_{3}-B_{4}\right) B_{5} B_{6}\left(b_{5}-b_{6}\right)-b_{3}^{2} b_{5}^{2}\left(B_{3}-B_{5}\right) B_{4} B_{6}\left(b_{4}-b_{6}\right)+b_{3}^{2} b_{6}^{2}\left(B_{3}-B_{6}\right) B_{4} B_{5}\left(b_{4}-b_{5}\right)+ \\
b_{4}^{2} b_{5}^{2}\left(B_{4}-B_{5}\right) B_{3} B_{6}\left(b_{3}-b_{6}\right)-b_{4}^{2} b_{6}^{2}\left(B_{4}-B_{6}\right) B_{3} B_{5}\left(b_{3}-b_{5}\right)+b_{5}^{2} b_{6}^{2}\left(B_{5}-B_{6}\right) B_{3} B_{4}\left(b_{3}-b_{4}\right)+ \\
b_{2}\left[B_{3}|\mathbf{b}, \mathbf{B}, \mathbf{B b}|_{4}^{6}-B_{4}|\mathbf{b}, \mathbf{B}, \mathbf{B b}|_{(3,5,6)}+B_{5}|\mathbf{b}, \mathbf{B}, \mathbf{B b}|_{(3,4,6)}-B_{6}|\mathbf{b}, \mathbf{B}, \mathbf{B b}|_{3}^{5}\right]=0 .
\end{array}
$$

2. We set $b_{2}=0$, compute $B_{5}$ from $L_{2}=0$ and $a_{5}$ fom $L_{3}=0$.

a. $B_{6}=b_{6}=0$.

b. We compute $B_{6}$ from $K_{2}$ and one equation remains:

$$
\begin{array}{r}
A_{2} B_{3}\left[a_{6} b_{4}\left(b_{3}-b_{6}\right)-a_{4} b_{3} b_{6}\right]-A_{2} B_{4}\left[a_{6} b_{3}\left(b_{4}-b_{6}\right)-a_{3} b_{4} b_{6}\right] \\
+b_{6}^{2}|\mathbf{a}, \mathbf{A}, \mathbf{B}|_{2}^{4}+a_{2}\left(b_{3} b_{4}-b_{4} b_{6}-b_{3} b_{6}\right)|\mathbf{A}, \mathbf{B}|_{3}^{4}=0
\end{array}
$$

Proof: We split the proof up into two parts:

Part [A] In this part we show that $K_{1}=K_{2}=K_{4}=L_{1}=L_{2}=L_{3}=0$ are the six necessary and sufficient conditions for a non-architecturally singular manipulator with no four anchor points collinear to be Schönflies-singular.

We set $e_{1}=e_{2}=0(\Rightarrow$ axis a equals the $z$-axis of the fixed system $)$ and compute $Q$. The necessity of $K_{1}=K_{2}=K_{4}=0$ follows from:

$$
Q_{002}^{60}=K_{1}, \quad Q_{101}^{60}=K_{2}, \quad Q_{001}^{80}-Q_{001}^{08}=2 A_{2} K_{4} .
$$

Due to Lemma 2 we can perform the elementary operations with the matrix Q given by Karger [4] without loss of generality (w.l.o.g.). These five steps can be written as follows:

(a) $\underline{\mathbf{l}}_{i}:=\underline{\mathbf{l}}_{i}-\underline{\mathbf{l}}_{1} \quad i=2, \ldots, 6$

(c) $\underline{\mathbf{l}}_{i}:=\underline{\mathbf{l}}_{i}-\underline{\mathbf{l}}_{3} \frac{|\mathbf{A}, \mathbf{B}|_{(2, i)}}{|\mathbf{A}, \mathbf{B}|_{2}^{3}} \quad i=4,5,6$

(e) $\underline{\mathbf{l}}_{6}:=\underline{\mathbf{l}}_{6}-\underline{\mathbf{l}}_{5} \frac{|\mathbf{A}, \mathbf{B}, \mathbf{B a}, \mathbf{B b}|_{(2,3,4,6)}}{|\mathbf{A}, \mathbf{B}, \mathbf{B a}, \mathbf{B b}|_{2}^{5}}$. (b) $\underline{\mathbf{l}}_{i}:=\underline{\mathbf{l}}_{i}-\underline{\mathbf{l}}_{2} A_{i} / A_{2} \quad i=3, \ldots, 6$

(d) $\underline{\mathbf{l}}_{i}:=\underline{\mathbf{l}}_{i}-\underline{\mathbf{l}}_{4} \frac{|\mathbf{A}, \mathbf{B}, \mathbf{B a}|_{(2,3, i)}}{|\mathbf{A}, \mathbf{B}, \mathbf{B a}|_{2}^{4}} \quad i=5,6$

Finally $\underline{\mathbf{l}}_{6}$ has the following structure:

$\left(r_{11} K_{1}+r_{13} K_{2}, r_{21} K_{1}+r_{23} K_{2}, r_{31} K_{1}+r_{33} K_{2}, 0,-r_{31} K_{3}-r_{33} K_{4}, r_{21} K_{3}+r_{23} K_{4}\right)$

with $K_{i}(i=1, \ldots, 4)$ of Eq. (4) and $r_{i j}$ of Eq. (1). ${ }^{2}$ Due to Eq. (7) we set $K_{1}=K_{2}=K_{4}=0$ in Eq. (8) and compute $Q=A_{2} e_{0} e_{3} K_{3} F$ [744]. The

$\overline{2}$ Note that $K_{1}=K_{2}=K_{3}=K_{4}=0$ are the generalized version $\left(b_{2} \neq 0\right)$ of the 4 sufficient and necessary conditions for a planar parallel manipulators with no 4 points collinear to be architecturally singular (cf. [4]). 
necessity of the remaining conditions follows from:

$F_{002}^{40}=|\mathbf{A}, \mathbf{B}, \mathbf{B b}, \mathbf{a}|_{2}^{5}, \quad F_{101}^{40}=|\mathbf{A}, \mathbf{B}, \mathbf{B b}, \mathbf{b}|_{2}^{5}, \quad F_{001}^{60}+F_{001}^{06}=2|\mathbf{A}, \mathbf{B}, \mathbf{B b}, \mathbf{A b}|_{2}^{5}$.

For the proof of the sufficiency we must show that $F[744]$ vanishes if these 3 conditions are fulfilled. A close inspection of the coefficients of $F$ shows that they all can be written as determinants of $4 \times 4$ submatrices of the matrix $\mathbf{S}:=(\mathbf{A}, \mathbf{B}, \mathbf{B b}, \mathbf{a}, \mathbf{b}, \mathbf{A b})_{2}^{5}$ or linear combinations of those. But the conditions of Eq. (9) imply $r k(\mathbf{S}) \leq 3$ because $\mathbf{A}_{2}^{5}, \mathbf{B}_{2}^{5}, \mathbf{B b}_{2}^{5}$ are linearly independent due to Lemma 2. This finishes the first part.

Part [B] In this part we show that the only non-architecturally singular manipulators with no 4 points collinear fulfilling $K_{1}=K_{2}=K_{4}=L_{1}=L_{2}=$ $L_{3}=0$ are the listed 3 designs.

Due to Lemma 2 we can compute $A_{6}$ from $K_{1}=0$ and $A_{5}$ from $L_{1}=0$ w.l.o.g.. In the following we distinguish two cases:

1. $b_{2} \neq 0$ : Under this assumption we can express $a_{6}$ from $K_{2}=0$ and $a_{5}$ from $L_{2}=0$.

a. $\left(b_{3}-b_{5}\right)\left(b_{4}-b_{5}\right) \neq 0$ : Now we can also express $A_{4}$ from $L_{3}=0$. Then $K_{4}=0$ can only vanish without contradiction (w.c.) if the condition Eq. (5) is fulfilled. This yields the solution of item 1.

b. $b_{i}=b_{5}$ with $i, j \in\{3,4\}$ and $i \neq j$ : Now $L_{3}=0$ can only vanish w.c. for $b_{5}\left(b_{5}-b_{2}\right)=0$.

i. $b_{5}=0$ : Now $K_{4}=0$ can only vanish w.c. for

$$
A_{2} B_{i} b_{j}\left(b_{2}-b_{6}\right)\left(B_{j}-B_{6}\right)+\left(A_{j} B_{i}-A_{i} B_{j}\right) b_{2} B_{6}\left(b_{j}-b_{6}\right)=0 .
$$

It should be noted that $\mathrm{m}_{1}, \mathrm{~m}_{i}, \mathrm{~m}_{5}$ and $\mathrm{M}_{1}, \mathrm{M}_{i}, \mathrm{M}_{5}$ are collinear. This solution corresponds with item $2 \mathrm{a}$.

ii. $b_{5}=b_{2}$ : This can be done analogously to the above case. Again the solution corresponds with item $2 \mathrm{a}$.

2. $b_{2}=0$ : Now $L_{2}=0$ can only vanish w.c. for $|\mathbf{b}, \mathbf{B}, \mathbf{B b}|_{3}^{5}=0$. It is very easy to verify that this equation cannot be solved for $B_{3}, B_{4}$ or $B_{5}$ if 4 points are collinear or $|\mathbf{A}, \mathbf{B}, \mathbf{B a}, \mathbf{B b}|_{2}^{5}=0$. Therefore we can assume w.l.o.g. that $|\mathbf{b}, \mathbf{B}, \mathbf{B b}|_{3}^{5}=0$ can be solved for $B_{5}$. As a consequence we can also express $a_{5}$ from $L_{3}=0$ w.l.o.g..

Now $K_{2}=0$ can only vanish w.c. for $|\mathbf{b}, \mathbf{B}, \mathbf{B b}|_{(3,4,6)}=0$. Again it can easily be shown that this equation cannot be solved for $B_{3}, B_{4}$ or $B_{6}$ w.c. only for $b_{6}=B_{6}=0, B_{3}=B_{4}$. But this is a special solution of item 2a.

For the general case we can assume w.l.o.g. that $B_{6}$ can be expressed from $|\mathbf{b}, \mathbf{B}, \mathbf{B b}|_{(3,4,6)}=0$. Now $K_{4}=0$ can only vanish w.c. for $b_{6}=0$ which yield item $2 \mathrm{a}$ or if Eq. (6) is fulfilled. The latter case yields item $2 \mathrm{~b}$. 


\section{Four base anchor points are collinear}

W.l.o.g. we can assume $\mathrm{M}_{1}, \ldots, \mathrm{M}_{4}$ are collinear. Now we can choose Cartesian coordinate systems such that $A_{1}=B_{1}=B_{2}=B_{3}=B_{4}=a_{1}=b_{1}=0$ hold. In the first step we show that $\mathrm{m}_{5}=\mathrm{m}_{6}$ or $B_{5} B_{6}=0$ yield architecturally singular manipulators:

1. For $B_{5}=0$ we get $Q=B_{6}\left(z+b_{6} K\right) F[384]$. Inspection of the coefficients of $F$ show that the condition $r k(\mathbf{A}, \mathbf{a}, \mathbf{b}, \mathbf{A a}, \mathbf{A b})_{2}^{5} \leq 3$ must hold because the determinants of all five possible $4 \times 4$ submatrices appear as coefficients or can be produced as linear combinations of coefficients. According to $[19,15]$ this already implies an architecturally singular design.

2. $\mathrm{m}_{5}=\mathrm{m}_{6}$ : Now $Q$ splits up into $\left(z+b_{6} K\right) F[768]$. The conditions

$$
\begin{aligned}
F_{020}^{40} & =|\mathbf{A}, \mathbf{B}, \mathbf{A b}, \mathbf{a}, \mathbf{b}|_{2}^{6}=0, & & F_{100}^{42}=|\mathbf{A}, \mathbf{B}, \mathbf{A} \mathbf{A}, \mathbf{a}, \mathbf{b}|_{2}^{6}=0, \\
F_{010}^{51}+F_{010}^{15} & =|\mathbf{A}, \mathbf{B}, \mathbf{A a}, \mathbf{A b}, \mathbf{b}|_{2}^{6}=0, & F_{001}^{42} & =|\mathbf{A}, \mathbf{B}, \mathbf{A a}, \mathbf{A b}, \mathbf{a}|_{2}^{6}=0,
\end{aligned}
$$

are the generalized version $\left(b_{2} \neq 0\right)$ of those given by Nawratil [14], indicating the degenerated cases of architecturally singular planar manipulators.

Theorem 2 For a planar Stewart Gough platform with 4 collinear base anchor points we can assume Cartesian coordinate systems such that $A_{1}=B_{1}=$ $B_{2}=B_{3}=B_{4}=a_{1}=b_{1}=0$ hold. Then a non-architecturally singular manipulator, where a is orthogonal to $\Phi$ and parallel to $\varphi$, is $\mathrm{X}(\mathrm{a})$-singular in one of the following cases (after permutation of indices):

1. $\underline{\left[\mathrm{m}_{5}, \mathrm{~m}_{6}\right] \| \Phi:}$

$\overline{\text { a. Compute }} A_{4}$ from $|\mathbf{A}, \mathbf{a}, \mathbf{b}|_{2}^{4}=0, A_{3}$ from $|\mathbf{A b}, \mathbf{a}, \mathbf{b}|_{2}^{4}=0$ and $A_{6}$ from $|\mathbf{A} \mathbf{b}, \mathbf{B}, \mathbf{a}, \mathbf{b}|_{3}^{6}=0$. The condition $|\mathbf{A}, \mathbf{B}, \mathbf{a}, \mathbf{b}|_{3}^{6} /\left(A_{2} c_{(1,3,4)}\right)=0$ remains.

b. $\mathbf{M}_{1}, \mathrm{M}_{5}, \mathrm{M}_{6}$ collinear: Compute $A_{4}$ from $|\mathbf{A}, \mathbf{a}, \mathbf{b}|_{2}^{4}=0$.

i. $\mathrm{m}_{2}, \mathrm{~m}_{3}, \mathrm{~m}_{4}$ are situated on a line which is parallel to $\Phi$, condition $|\mathbf{A}, \mathbf{B}, \mathbf{a}, \mathbf{b}|_{(2,3,5,6)}=0$ remains.

ii. $\left[\mathrm{m}_{3}, \mathrm{~m}_{4}\right] \| \Phi, \mathrm{M}_{1}=\mathrm{M}_{2}$, condition $|\mathbf{B}, \mathbf{a}, \mathbf{b}|_{(2,5,6)}=0$ remains.

c. $\mathrm{m}_{1}, \mathrm{~m}_{2}, \mathrm{~m}_{3}, \mathrm{~m}_{4}$ are situated on a line which is parallel to $\Phi$ and $\mathrm{M}_{5}=\mathrm{M}_{6}$.

2. $\mathrm{m}_{1}, \mathrm{~m}_{5}, \mathrm{~m}_{6}$ are situated on a line which is parallel to $\Phi$ :

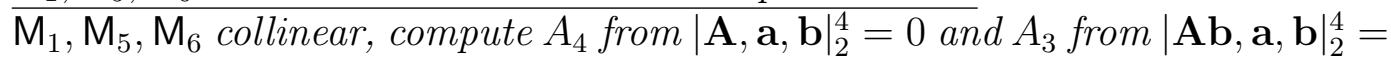
0 , condition $|\mathbf{a}, \mathbf{B}|_{5}^{6}=0$ remains.

3. $\mathrm{m}_{1}, \mathrm{~m}_{2}, \mathrm{~m}_{5}, \mathrm{~m}_{6}$ are situated on a line which is parallel to $\Phi$ :

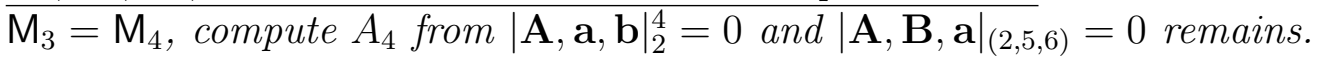

4. $\mathrm{m}_{1}, \mathrm{~m}_{3}, \mathrm{~m}_{4}, \mathrm{~m}_{5}, \mathrm{~m}_{6}$ are situated on a line which is parallel to $\Phi$ :

compute $A_{4}$ from $|\mathbf{A}, \mathbf{a}|_{3}^{4}=0$ and the condition $|\mathbf{A}, \mathbf{B}, \mathbf{a}|_{(3,5,6)}=0$ remains.

Proof: Due to $Q_{102}^{31}=B_{5} B_{6}\left(a_{5}-a_{6}\right)|\mathbf{A}, \mathbf{a}, \mathbf{b}|_{2}^{4}$ and $Q_{111}^{40}=B_{5} B_{6}\left(b_{5}-b_{6}\right)|\mathbf{A}, \mathbf{a}, \mathbf{b}|_{2}^{4}$ we set $|\mathbf{A}, \mathbf{a}, \mathbf{b}|_{2}^{4}=0$. The equation cannot be solved for any $A_{i}(i=2,3,4)$ if and only if $\mathrm{m}_{1}, \ldots, \mathrm{m}_{4}$ are collinear. 
- $\mathrm{m}_{1}, \ldots, \mathrm{m}_{4}$ collinear: We distinguish two cases:

$\star b_{2} \neq 0$ : We set $a_{i}=a_{2} b_{i} / b_{2}$ for $i=3,4$. Then $Q$ splits up into several factor. It can easily be seen that none of these factor can vanish without contradiction.

$\star b_{2}=b_{3}=b_{4}=0$ : Again $Q$ splits up into several factors, where the longest has 28 terms. This is the only factor which can vanish w.c. and it implies solution (1c).

- $\mathrm{m}_{1}, \ldots, \mathrm{m}_{4}$ not collinear: In this case we can relabel the points in such a way that $c_{(1,2,3)} \neq 0$ and $c_{(1,2,4)} \neq 0$ hold. We compute $A_{4}$ from $|\mathbf{A}, \mathbf{a}, \mathbf{b}|_{2}^{4}=0$. Now $Q_{110}^{60}=0$ and $Q_{011}^{60}+Q_{011}^{06}=0$ imply $|\mathbf{A b}, \mathbf{a}, \mathbf{b}|_{2}^{4}=0$.

1. $b_{3} \neq b_{4}$ : Under this assumption we can express $A_{3}$ from $|\mathbf{A b}, \mathbf{a}, \mathbf{b}|_{2}^{4}=0$. Then $Q_{002}^{51}=0$ and $Q_{101}^{51}+Q_{101}^{15}=0$ can only vanish w.c. in two cases:

a. $b_{5}=b_{6}$. Now $Q$ splits up into several factors; one of them $F$ has 480 terms. The vanishing of all other factors yield easy contradictions.

i. $b_{6} \neq 0$ : W.l.o.g. we can compute $A_{6}$ from $F_{100}^{31}=|\mathbf{A b}, \mathbf{B}, \mathbf{a}, \mathbf{b}|_{3}^{6}=0$. Now the condition $F_{011}^{20}=|\mathbf{A}, \mathbf{B}, \mathbf{a}, \mathbf{b}|_{3}^{6}=0$ remains. This condition splits up into $A_{2} c_{(1,3,4)} G[48]=0$. Then $A_{2} c_{(1,3,4)}=0$ yields a contradiction and from $G[48]=0$ we get solution (1a).

ii. $b_{6}=0$ : Now the two conditions $F_{010}^{40}+F_{010}^{04}=|\mathbf{A b}, \mathbf{A}, \mathbf{B}, \mathbf{b}|_{3}^{6}=0$ and $F_{100}^{31}=0$ can only vanish w.c. in the following 3 cases:

$\star A_{6}=B_{6} A_{5} / B_{5}$ and $a_{6}=B_{6} a_{5} / B_{5}$, which yields solution (2).

$\star$ For $b_{2}=0$ the remaining coefficients of $F$ can only vanish w.c. for $|\mathbf{a}, \mathbf{A}, \mathbf{B}|_{(2,5,6)}=0$ which yields solution (3).

$\star$ For $b_{i}=0$ the remaining coefficients of $F$ can only vanish w.c. for $|\mathbf{A}, \mathbf{B}, \mathbf{a}, \mathbf{b}|_{(2, j, 5,6)}=0$ with $i \neq j$ and $i, j \in\{3,4\}$. We get solution (3) after a permutation of indices.

b. $|\mathbf{a}, \mathbf{b}, \mathbf{b b}|_{2}^{4}=0,|\mathbf{a}, \mathbf{b}, \mathbf{a b}|_{2}^{4}=0, b_{5} \neq b_{6}$ : The resultant of these conditions with respect to $a_{4}$ can only vanish w.c. for $b_{4}=0$ or $b_{2}=b_{4}$. Plugging these conditions into the above two expressions already yields a contradiction.

2. $b_{3}=b_{4}$ : Now $|\mathbf{A b}, \mathbf{a}, \mathbf{b}|_{2}^{4}=0$ splits up into $A_{2} b_{4}\left(b_{2}-b_{4}\right)\left(a_{3}-a_{4}\right)=0$. As for $a_{3}=a_{4}$ the $3^{r d}$ and $4^{\text {th }}$ leg coincide, we must distinguish three cases:

a. $b_{4}=0$ : In this case $\mathrm{m}_{1}, \mathrm{~m}_{3}, \mathrm{~m}_{4}$ are collinear. Now $Q_{101}^{51}+Q_{101}^{15}=0$ implies $b_{5}=b_{6}$ and $Q$ splits up into several factor where only the one with 68 terms yields no contradiction. It can easily be seen that the coefficients of this factor can only vanish in the following two cases:

i. $b_{6}=0$ and $|\mathbf{a}, \mathbf{A}, \mathbf{B}|_{(3,5,6)}=0$ yield solution (4).

ii. $C_{(2,5,6)}=0$ and $|\mathbf{a}, \mathbf{b}, \mathbf{A}, \mathbf{B}|_{(2,3,5,6)}=0$ yield solution (1bi) after a permutation of indices.

b. $b_{2}=b_{4} \neq 0$ : This case is similar to the last one. After performing the same steps as above we end up with the following two solutions:

i. $b_{4}=b_{5}=b_{6}$ and $|\mathbf{a}, \mathbf{b}, \mathbf{A}, \mathbf{B}|_{(2,3,5,6)}=0$ yield solution (4) after a 
permutation of indices.

ii. $b_{5}=b_{6}, C_{(1,5,6)}=0$ and $|\mathbf{a}, \mathbf{b}, \mathbf{A}, \mathbf{B}|_{(2,3,5,6)}=0$ yield solution (1bi).

c. $A_{2}=0, b_{4}\left(b_{2}-b_{4}\right) \neq 0$ : Now $Q_{002}^{51}=0$ and $Q_{101}^{51}+Q_{101}^{15}=0$ imply again $b_{5}=b_{6}$. Now it can easily be seen that the other conditions can only vanish w.c. in the following two cases:

i. $b_{4}=b_{6}$ and $|\mathbf{a}, \mathbf{b}, \mathbf{A}, \mathbf{B}|_{(2,3,5,6)}=0$ yield solution (3) after a permutation of indices.

ii. $C_{(1,5,6)}=0$ and $|\mathbf{a}, \mathbf{b}, \mathbf{B}|_{(2,5,6)}=0$ yield solution (1bii).

\section{Four platform anchor points are collinear}

Theorem 3 For a planar Stewart Gough platform with 4 collinear platform anchor points parallel to $\Phi$ and no 4 collinear base anchor points we can assume Cartesian coordinate systems such that $A_{1}=B_{1}=B_{2}=a_{1}=b_{1}=b_{2}=$ $b_{3}=b_{4}=0$ hold. Then a non-architecturally singular manipulator, where a is orthogonal to $\Phi$ and parallel to $\varphi$, is $\mathrm{X}(\mathrm{a})$-singular in one the following cases (after permutation of indices):

1. $\mathrm{m}_{1}, \ldots, \mathrm{m}_{5}$ are situated on a line which is parallel to $\Phi$ :

a. Compute $a_{3}$ from $|\mathbf{A}, \mathbf{B}, \mathbf{a}|_{3}^{5}=0$ and $|\mathbf{A}, \mathbf{B}, \mathbf{a}|_{(2,4,5)}=0$ remains.

b. $\mathrm{m}_{1}=\mathrm{m}_{2}$, compute $a_{3}$ from $|\mathbf{a}, \mathbf{B}|_{3}^{4}=0$ and $|\mathbf{a}, \mathbf{B}|_{4}^{5}=0$ remains.

2. $\mathrm{m}_{1}, \ldots, \mathrm{m}_{4}$ are situated on a line which is parallel to $\Phi$ and $\mathrm{M}_{5}=\mathrm{M}_{6}$ :

a. Compute $A_{4}$ from $|\mathbf{A}, \mathbf{B}, \mathbf{a}|_{2}^{4}=0$ and $|\mathbf{A}, \mathbf{B}, \mathbf{a}, \mathbf{b}|_{(2,3,5,6)}=0$ remains.

b. $\mathrm{m}_{1}=\mathrm{m}_{2}$, compute $a_{3}$ from $|\mathbf{a}, \mathbf{B}|_{3}^{4}=0$ and $|\mathbf{a}, \mathbf{b}, \mathbf{B}|_{4}^{6}=0$ remains.

Proof: We split the proof again into two parts:

Part [A] We assume that $\mathrm{m}_{1}, \ldots, \mathrm{m}_{5}$ are collinear; i.e. $b_{5}=0$. Now $Q$ splits up into

$$
z^{2} b_{6}\left(2 e_{0} e_{3}\left(x-A_{6} K\right)-\left(e_{0}^{2}-e_{3}^{2}\right)\left(y-B_{6} K\right)\right) F[42]
$$

and two conditions $\left(F^{20}=0\right.$ and $\left.F^{11}=0\right)$ remain. Computing the resultant of these two conditions with respect to $A_{2}$ yields $a_{2}|\mathbf{A}, \mathbf{B}, \mathbf{a}|_{3}^{5} G[12]$. As $G=0$ and $F^{20}=0$ imply item 10 of Karger's list of architecturally singular manipulators given in Theorem 3 of [6], there are two possibilities left:

1. $a_{2}=0$ : In this case the two equations $F^{20}=A_{2}|\mathbf{A}, \mathbf{B a}, \mathbf{a}|_{3}^{5}=0$ and $F^{11}=A_{2}|\mathbf{B}, \mathbf{B a}, \mathbf{a}|_{3}^{5}=0$ remain. The resultant with respect to $a_{5}$ yields $A_{2}^{2} a_{3} a_{4} B_{5} C_{(3,4,5)}|\mathbf{a}, \mathbf{B}|_{3}^{4}$. As $C_{(3,4,5)}=0$ implies the special case of item 10 of Karger's list, we are left with three cases:

a. $a_{3} a_{4}=0$ : W.l.o.g. we set $a_{3}=0$. Then $F^{20}=0$ imply $B_{4}=B_{5}$ and $F^{11}=0$ yields the contradiction.

b. $B_{5}=0, a_{3} a_{4} \neq 0$ : From $F^{20}=0$ we get $a_{3}=a_{4}$. Then $F^{11}=0$ yields the contradiction. 
c. $|\mathbf{a}, \mathbf{B}|_{3}^{4}=0, a_{3} a_{4} B_{5} \neq 0$ : Now $F^{20}=0$ and $F^{11}=0$ can only vanish w.c. for $|\mathbf{a}, \mathbf{B}|_{4}^{5}=0$. This yields solution (1b).

2. $|\mathbf{A}, \mathbf{B}, \mathbf{a}|_{3}^{5}=0, a_{2} \neq 0$ : As $C_{(1,3,4)}=C_{(1,3,5)}=C_{(1,4,5)}=0$ yields a contradiction we can assume w.l.o.g. that $C_{(1,4,5)} \neq 0$ holds. Therefore we can express $a_{3}$ from $|\mathbf{A}, \mathbf{B}, \mathbf{a}|_{3}^{5}=0$. Then we get $F^{20}=|\mathbf{A}, \mathbf{B}, \mathbf{a}|_{(2,4,5)} H_{1}[8]$ and $F^{11}=|\mathbf{A}, \mathbf{B}, \mathbf{a}|_{(2,4,5)} H_{2}[8]$. The vanishing of the common factor yields solution (1a).

The resultant of the remaining two factors with respect to $A_{3}$ yields $B_{3} a_{4} a_{5}\left(B_{3}-B_{4}\right)\left(B_{3}-B_{5}\right)\left(a_{4}-a_{5}\right)|\mathbf{A}, \mathbf{B}|_{4}^{5}$. All possible cases yield easy contradictions after back-substitution into $H_{1}=0$ and $H_{2}=0$.

Part [B] Only 4 platform anchor points are collinear. Now $Q$ splits up into $z F[1028]$. Due to $F_{200}^{31}=0$ and $F_{020}^{40}=0$ we must distinguish the following two cases:

1. $\mathrm{M}_{5}=\mathrm{M}_{6}: F$ splits up into $z\left(2 e_{0} e_{3}\left(x-A_{6} K\right)-\left(e_{0}^{2}-e_{3}^{2}\right)\left(y-B_{6} K\right)\right) R[84]$ and two conditions $\left(R^{20}=0\right.$ and $\left.R^{11}=0\right)$ remain. These are exactly the two conditions given in Eq. (18) by Karger [6]. But it is shown in [14] that these conditions are not sufficient for an architecturally singular design. There are three cases where these two conditions vanish and the manipulator is not architecturally singular. Two of these cases correspond to solution (2a) and $(2 \mathrm{~b})$, respectively. The third case equals case (1c) of Theorem 2 .

2. $|\mathbf{A}, \mathbf{B}, \mathbf{a}|_{2}^{4}=0, \mathrm{M}_{5} \neq \mathrm{M}_{6}$ : As no 4 base anchor points are collinear we can assume $A_{2} B_{3} \neq 0$ w.l.o.g. . Therefore we can express $a_{4}$ from $|\mathbf{A}, \mathbf{B}, \mathbf{a}|_{2}^{4}=0$. Then $F$ splits up into $S[14] T[120]$. It can easily be seen that $S^{20}=0$ and $S^{11}=0$ as well as $T_{100}^{40}=b_{5} b_{6} A_{2} B_{3}\left(B_{5}-B_{6}\right)=0$ and $T_{010}^{40}+T_{010}^{04}=$ $2 b_{5} b_{6} A_{2} B_{3}\left(A_{5}-A_{6}\right)=0$ imply contradictions.

Note that item (3) of Theorem 2 is a special case of item (2a) of Theorem 3. Moreover, item (4) of Theorem 2 is a special case of item (1a) of Theorem 3. Therefore the remarkable set of Schönflies-singular planar parallel manipulators contains 12 different manipulator designs.

Remark 1 It should also be mentioned that the only degenerated manipulators are those given in item (1a) and (1b) of Theorem 3, because they are independent of the choice of the sixth platform and base anchor point; i.e. the first five legs always belong to a congruence of lines. Therefore these designs also yield non-planar Schönflies-singular manipulators which are not architecturally singular.

Theorem 4 For a planar Stewart Gough platform with 4 collinear platform anchor points and no 4 collinear base anchor points we can assume Cartesian coordinate systems such that $A_{1}=B_{1}=B_{2}=a_{1}=b_{1}=0$ hold. If no 4 collinear platform anchor points are parallel to $\Phi$, then there does not exist any $\mathrm{X}(\mathrm{a})$-singular manipulator, where a is orthogonal to $\Phi$ and parallel to $\varphi$. 
Proof: This proof of the non-existence can be done as in Theorem 2 and 3, respectively. For the lengthy discussion of cases we refer to the appendix of this technical report.

Remark 2 At the end of this case study we want to recall that the manipulators given in Theorem 1, 2 and 3 are also Schönflies-singular for any $\beta \in[0, \pi / 2]$ due to Lemma 1. But the set of non-architecturally singular manipulators which are $\mathrm{X}(\mathrm{a})$-singular with a orthogonal to $\Phi$ and $\varphi$ is much larger than the set of manipulators obtained from Theorem 1, 2 and 3 by setting $\beta=\pi / 2$. For more details we refer to [17].

\section{Geometric characterization}

Lemma 3 The Schönflies-singular planar parallel manipulators given in Theorem 1, 2 and 3 fulfill the following rank condition:

$$
r k(\mathbf{1}, \mathbf{A}, \mathbf{B}, \mathbf{B b}, \mathbf{a}, \mathbf{b}, \mathbf{A b})_{1}^{6}=4 .
$$

Proof:

ad Theorem 1: $K_{1}=K_{2}=K_{4}=0$ imply $r k(\mathbf{1}, \mathbf{A}, \mathbf{B}, \mathbf{B b}, \mathbf{a}, \mathbf{b}, \mathbf{A b}, \mathbf{B a})_{1}^{6} \leq 5$ because $\mathbf{A}, \mathbf{B}, \mathbf{B a}, \mathbf{B b}$ are linearly independent due to Lemma 2. From the proof of Theorem 1 we already know $r k(\mathbf{1}, \mathbf{A}, \mathbf{B}, \mathbf{B b}, \mathbf{a}, \mathbf{b}, \mathbf{A b})_{1}^{5} \leq 4$. This already implies the condition given above because if the rank is smaller than 4 the manipulator is architecturally singular according to $[19,15]$.

ad Theorem 2 and 3: For the special cases listed in these theorems the rankproperty can be proven explicitly.

In the following theorem we show the converse of this lemma:

Theorem 5 A non-architecturally singular Stewart Gough platform with planar base $\Phi$ and platform $\varphi$ which fulfills Eq. (12) and where a is orthogonal to $\Phi$ and orthogonal to the $x$-axis of the moving frame is $\mathrm{X}(\mathrm{a})$-singular.

Proof: This geometric proof is done according to the method introduced by Röschel and Mick [19]. For readers who are not familiar with line geometry we refer to [18].

All lines of a linear line complex $\mathcal{C}$ with homogeneous coordinates $\left(c_{1}: \ldots: c_{6}\right)$ correspond with the null-lines of a null-polarity $\kappa$. This linear mapping $\kappa$ maps the point $\mathrm{P}$ with homogeneous coordinates $\left(p_{0}: \ldots: p_{3}\right)$ onto the plane $\kappa(\mathrm{P})$ 
with homogeneous coordinates $\left[\xi_{0}: \ldots: \xi_{3}\right]$ by

$$
\left(\begin{array}{l}
\xi_{0} \\
\xi_{1} \\
\xi_{2} \\
\xi_{3}
\end{array}\right)=\left(\begin{array}{cccc}
0 & -c_{4} & -c_{5} & -c_{6} \\
c_{4} & 0 & -c_{3} & c_{2} \\
c_{5} & c_{3} & 0 & -c_{1} \\
c_{6} & -c_{2} & c_{1} & 0
\end{array}\right)\left(\begin{array}{l}
p_{0} \\
p_{1} \\
p_{2} \\
p_{3}
\end{array}\right) .
$$

If we restrict $\kappa$ to the points $\mathrm{M}_{i}$ of the base $\Phi$ and intersect $\kappa\left(\mathrm{M}_{i}\right)$ with the platform $\varphi$ we get a correlation $\gamma$ from points of $\Phi$ to lines of $\varphi$. Due to Lemma 1 we can assume that $\varphi$ is parallel to a. Now the platform anchor points $\mathbf{M}_{i}$ with homogeneous coordinates $\left(1: A_{i}: B_{i}: 0\right)$ and base anchor points $\mathrm{m}_{i}$ with $\left(1: a_{i}: 0: b_{i}\right)$ are conjugate points with respect to $\gamma$ if

$$
\left(1, a_{i}, b_{i}\right)\left(\begin{array}{ccc}
0 & -c_{4} & -c_{5} \\
c_{4} & 0 & -c_{3} \\
c_{6} & -c_{2} & c_{1}
\end{array}\right)\left(\begin{array}{c}
1 \\
A_{i} \\
B_{i}
\end{array}\right)=0
$$

holds. Moreover this condition must hold for the whole Schönflies group X(a) where a is orthogonal to $\Phi$ and parallel to $\varphi$. Therefore Eq. (14) must hold independently of translations of $\Phi$ in $x$ and $y$ direction as well as rotations about the $z$-axis (angle $\delta$ ) and independently of translations of $\varphi$ in $z$ direction. This yields $\left(1, a_{i}, b_{i}\right) \mathbf{A}\left(1, A_{i}, B_{i}\right)^{T}=0$ with

$$
\mathbf{A}:=\left(a_{i j}\right)=\left(\begin{array}{lll}
1 & 0 & z \\
0 & 1 & 0 \\
0 & 0 & 1
\end{array}\right)\left(\begin{array}{ccc}
0 & -c_{4} & -c_{5} \\
c_{4} & 0 & -c_{3} \\
c_{6} & -c_{2} & c_{1}
\end{array}\right)\left(\begin{array}{ccc}
1 & 0 & 0 \\
x & \cos \delta & -\sin \delta \\
y & \sin \delta & \cos \delta
\end{array}\right) .
$$

The $a_{i j}$ are homogeneous linear functions of the coordinates $c_{i}$. Therefore the set of linear line complexes spanned by Schönflies-singular manipulators determine a 4-parametric manifold (parameters $x, y, z, \delta$ ) of correlations. Moreover the three equation $a_{00}=0, a_{11}=0$ and $a_{01}+a_{10}=0$ must hold. As $a_{11}$ of Eq. (15) equals $-c_{3} \sin \delta$ the condition $a_{11}=0$ implies $c_{3}=0$. As a consequence $a_{12}=-c_{3} \cos \delta$ is also zero. Therefore the ideal points of $\Phi$ are mapped onto the pencil of lines through the ideal point of the $x$-axis of the moving frame.

The remaining two conditions $a_{00}=0$ and $a_{01}+a_{10}=0$ can be written as

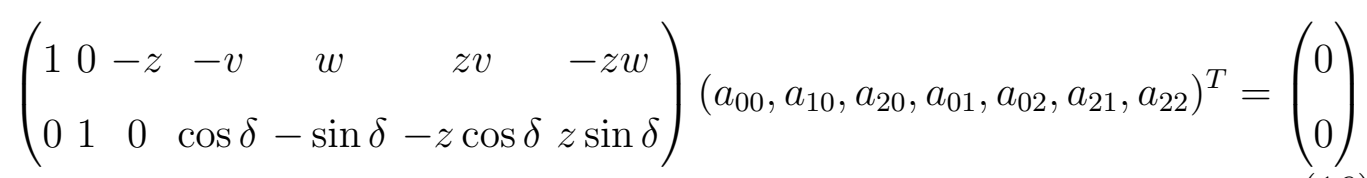


with $v:=x \cos \delta+y \sin \delta$ and $w:=x \sin \delta-y \cos \delta$. Due to the first two columns this $2 \times 7$ matrix has rank 2 independently of the parameters $x, y, z, \delta$. Moreover we can also rewrite the 6 equations $\left(1, a_{i}, b_{i}\right) \mathbf{A}\left(1, A_{i}, B_{i}\right)^{T}=0(i=1, \ldots, 6)$ in an analogous form as

$$
\left(\begin{array}{ccccccc}
1 & a_{1} & b_{1} & A_{1} & B_{1} & A_{1} b_{1} & B_{1} b_{1} \\
\vdots & \vdots & \vdots & \vdots & \vdots & \vdots & \vdots \\
1 & a_{6} & b_{6} & A_{6} & B_{6} & A_{6} b_{6} & B_{6} b_{6}
\end{array}\right)\left(a_{00}, a_{10}, a_{20}, a_{01}, a_{02}, a_{21}, a_{22}\right)^{T}=\left(\begin{array}{c}
0 \\
\vdots \\
0
\end{array}\right)
$$

If this $6 \times 7$ matrix has rank 4 the system of linear equations given in Eq. (16) and (17) has at least a 1-dimensional solution. As a consequence the 6parametric linear manifold of correlations described by (3,3)-matrices $a_{i j}$ with $a_{11}=a_{12}=0$ contains at least one correlation $\gamma$ and therefore the manipulator is Schönflies-singular.

This geometric proof also provides us the following geometric characterization:

Corrolary 1 Given are two sets of points $\left\{\mathrm{M}_{i}\right\}$ and $\left\{\mathrm{m}_{i}\right\}(i=1, \ldots, 6)$ in two planes $\Phi$ and $\varphi$, respectively. Then the non-architecturally singular planar parallel manipulator of Stewart Gough type, where a is orthogonal to $\Phi$ and parallel to $\varphi$, is $\mathrm{X}(\mathrm{a})$-singular if and only if $\left\{\mathrm{M}_{i}, \mathrm{~m}_{i}\right\}$ are three-fold conjugate pairs of points with respect to a 2-dimensional linear manifold of correlations, which map the ideal points of $\Phi$ onto the pencil of lines through the ideal point of the intersection line of $\varphi$ and $\Phi$.

Based on the results of this section we can also prove the following theorem:

Theorem 6 A non-architecturally singular planar manipulator which is $\mathrm{X}(\mathrm{a})$ singular with a orthogonal to $\Phi$ and parallel to $\varphi$ has a quadratic singularity surface.

Proof: First of all we show that $r k(\mathbf{D})=4$ with $\mathbf{D}:=(\mathbf{1}, \mathbf{a}, \mathbf{b}, \mathbf{A}, \mathbf{B})_{1}^{6}$ holds. The proof is done by contradiction as follows:

The relation between the rank of the matrix $\mathbf{D}$ and the geometry of the planar parallel manipulator was studied by Karger [8]. In the trivial cases $r k(\mathbf{D})=1$ or $r k(\mathbf{D})=2$ we get architecturally singular manipulators as all points collapse into a point or onto a line, respectively. Moreover Karger proved that the rank of $\mathbf{D}$ is equal to 3 if and only if the base and the platform are affinely equivalent.

Now we assume that this is the case, i.e. $a_{i}=n_{11} A_{i}+n_{12} B_{i}$ and $b_{i}=n_{21} A_{i}+$ $n_{22} B_{i}$ for $i=1, \ldots, 6$. Then we compute $Q$ for $e_{1}=e_{2}=0$. It can easily be seen that $Q$ can only vanish if the affinity is singular or if the base anchor points are located on a conic section. Both possibilities yield again architecturally singular manipulators. Therefore $r k(\mathbf{D})=4$ must hold. 
Due to this result one of the following two linear combinations must hold:

$$
\begin{aligned}
& \text { (i) } B_{i} b_{i}=\lambda_{1} A_{i}+\mu_{1} B_{i}+\nu_{1} a_{i} \text {, } \\
& A_{i} b_{i}=\lambda_{2} A_{i}+\mu_{2} B_{i}+\nu_{2} a_{i}, \\
& \text { (ii) } B_{i} b_{i}=\lambda_{1} A_{i}+\mu_{1} B_{i}+\nu_{1} b_{i} \text {, } \\
& A_{i} b_{i}=\lambda_{2} A_{i}+\mu_{2} B_{i}+\nu_{2} b_{i}, \\
& b_{i}=\lambda_{3} A_{i}+\mu_{3} B_{i}+\nu_{3} a_{i}, \\
& a_{i}=\lambda_{3} A_{i}+\mu_{3} B_{i}+\nu_{3} b_{i},
\end{aligned}
$$

with $\lambda_{i}, \mu_{i}, \nu_{i} \in \mathbb{R}$. Moreover we can set $A_{1}=B_{1}=a_{1}=b_{1}=B_{2}=0$. As a consequence $B_{2} b_{2}$ is equal to zero, which yields $\lambda_{1} A_{2}+\nu_{1} a_{2}=0$ resp. $\lambda_{1} A_{2}+\nu_{1} b_{2}=0$. We can express $\lambda_{1}$ from this equation, because we can assume $A_{2} \neq 0$ without loss of generality. Now we substitute these expressions into the homogeneous line coordinates of $\underline{\mathbf{l}}_{i}$ and compute $Q$. It turns out that $Q$ splits up into three factors, where one is the orientational factor $e_{0} e_{2}-$ $e_{1} e_{3}$. The second factor only depends on the geometry and the third factor is homogeneous in the Euler parameters of degree 6. Moreover the last factor only depends quadratically on the translation parameters and therefore this class of manipulators possesses a quadratic singularity surface (cf. [5]).

\section{Self-motional behavior}

In this section we will investigate the set of Schönflies-singular manipulators of case (1a) with respect to self-motions within the 5 -dimensional manifold $\Gamma$ of configurations determined by $e_{0} e_{2}-e_{1} e_{3}=0$. As these manipulators are singular in each configuration of $\Gamma$ the necessary condition for a self-motion, namely to be singular in each pose of the motion, is trivially fulfilled. Therefore there is the hope of finding new self-motions of Stewart Gough platforms. For the already known self-motions we refer to [3,7] and the references therein.

For the determination of self-motions it is advantageous to work in the Study parameter space $P^{7}$ which is a 7 -dimensional projective space with homogeneous coordinates $e_{0}, \ldots, e_{3}, f_{0}, \ldots f_{3}$. We get this representation by substitution of the translation parameters $t_{1}, t_{2}, t_{3}$ in the formula above Eq. (1) by

$$
\begin{aligned}
& t_{1}=2\left(e_{0} f_{1}-e_{1} f_{0}+e_{2} f_{3}-e_{3} f_{2}\right), \quad t_{2}=2\left(e_{0} f_{2}-e_{2} f_{0}+e_{3} f_{1}-e_{1} f_{3}\right), \\
& t_{3}=2\left(e_{0} f_{3}-e_{3} f_{0}+e_{1} f_{2}-e_{2} f_{1}\right) .
\end{aligned}
$$

Now all points of $P^{7}$ which are located on the so called Study quadric $\Psi$ : $\sum_{i=0}^{3} e_{i} f_{i}=0$ correspond with an Euclidean displacement, with exception of the 3 -dimensional subspace $e_{0}=\ldots=e_{3}=0$ of $\Psi$ because these points cannot fulfill the normalizing condition $K=1$. It was shown by Husty [2] that the condition that $\mathbf{m}_{i}$ with coordinates $\mathbf{m}_{i}=\left(a_{i}, b_{i}, 0\right)$ is located on a sphere with center $\mathbf{M}_{i}$ with coordinates $\mathbf{M}_{i}=\left(A_{i}, B_{i}, 0\right)$ and radius $R_{i}$ can be expressed 
by the following homogeneous quadratic equation:

$$
\begin{aligned}
\Lambda_{i}: & F K+4 \sum_{i=0}^{3} f_{i}^{2}+2\left(e_{3}^{2}-e_{0}^{2}\right)\left(A_{i} a_{i}+B_{i} b_{i}\right)+2\left(e_{2}^{2}-e_{1}^{2}\right)\left(A_{i} a_{i}-B_{i} b_{i}\right)+ \\
& 4\left[\left(e_{0} f_{2}-f_{0} e_{2}\right)\left(B_{i}-b_{i}\right)-\left(e_{1} f_{3}-f_{1} e_{3}\right)\left(B_{i}-b_{i}\right)+\left(e_{2} f_{3}-f_{2} e_{3}\right)\left(A_{i}-a_{i}\right)\right. \\
& \left.+\left(e_{0} f_{1}-f_{0} e_{1}\right)\left(A_{i}-a_{i}\right)+e_{0} e_{3}\left(A_{i} b_{i}-B_{i} a_{i}\right)-e_{1} e_{2}\left(A_{i} b_{i}+B_{i} a_{i}\right)\right]=0
\end{aligned}
$$

with $F=A_{i}^{2}+B_{i}^{2}+a_{i}^{2}+b_{i}^{2}-R_{i}^{2}$.

We consider the variety $V$ spanned by $\Gamma, \Psi, \Lambda_{1}, \ldots, \Lambda_{6}$. In general the solution variety is empty, but for special geometries the variety can be $n$-dimensional. Cases with $n>0$ correspond to $n$-dimensional self-motions of the manipulator.

Note that Schönflies-singular manipulators with pure Schönflies self-motions can only be special cases of the list of parallel manipulators with Schönflies Borel-Bricard motions given by Borel [1]. The proof of the completeness of this list was given by Husty and Karger in [3]. Therefore we are only interested in Schönflies-singular manipulators with non pure Schönflies self-motions.

Moreover we can restrict ourselves to non-architecturally singular manipulators, because it was already shown in [13] that the rank condition

$$
r k(1, \mathbf{A}, \mathbf{B}, \mathbf{a}, \mathbf{b}, \mathbf{B a}, \mathbf{A a}, \mathbf{B b}, \mathbf{A b})_{1}^{6}<6
$$

of architecturally singular manipulators given in [19] implies the existence of a linear combination $\sum_{i=1}^{6} \Lambda_{i} \rho_{i}=0$ with $\rho_{i} \in \mathbb{R}$. Therefore all planar architecturally singular manipulators have self-motions.

Surprisingly the following result holds:

Theorem 7 The Schönflies-singular manipulators of Theorem 5 do not possess self-motions within the 5-dimensional manifold $\Gamma$ of configurations determined by $e_{0} e_{2}-e_{1} e_{3}=0$ which are not pure Schönflies motions.

Proof: In the first part we assume that $e_{0} \neq 0$. As the Study parameters are homogeneous we can normalize them such that $e_{0}=1$ holds. As a consequence we set $e_{2}=e_{1} e_{3}$. Moreover from $\Psi$ we get $f_{0}=-e_{1} f_{1}-e_{1} e_{3} f_{2}-e_{3} f_{3}$. According to Eq. (18)-(20) we substitute

$$
B_{i} b_{i}=\lambda_{1} A_{i}+\mu_{1} B_{i}+\nu_{1} t_{i}, \quad A_{i} b_{i}=\lambda_{2} A_{i}+\mu_{2} B_{i}+\nu_{2} t_{i}, \quad s_{i}=\lambda_{3} A_{i}+\mu_{3} B_{i}+\nu_{3} t_{i},
$$

with $s, t \in\{a, b\}$ and $s \neq t$ in $\Lambda_{i}$. We proceed by computing the equations $U_{i}:=\Lambda_{i}-\Lambda_{1}$ which only depend linear on $f_{1}, f_{2}, f_{3}$ for $i=2, \ldots, 6$. Now we consider the $5 \times 3$ coefficient matrix $\mathbf{M}$ of this system.

First we assume that $r k(\mathbf{M})<3$. This is the case if the determinant of all five 
$3 \times 3$ submatrices vanish. The determinant of the system $U_{i}, U_{j}, U_{k}$ is given by

$$
\left(e_{3}^{2}\left(\lambda_{3}-\nu_{3}\right)-2 \mu_{3} e_{3}-\left(\lambda_{3}+\nu_{3}\right)\right)|\mathbf{A}, \mathbf{B}, \mathbf{t}|_{(i, j, k)} .
$$

Therefore we must distinguish the following two cases:

- As there do not exist a Schönflies-singular manipulator with 5 collinear base anchor points we can assume that $\mathrm{M}_{1}, \mathrm{M}_{3}, \mathrm{M}_{4}$ are not collinear. Therefore we can express $s_{i}$ from the conditions $|\mathbf{A}, \mathbf{B}, \mathbf{t}|_{(3,4, i)}=0$ for $i=5,6$. Then one equation $|\mathbf{A}, \mathbf{B}, \mathbf{t}|_{2}^{4}=0$ remains. But the vanishing of this equation would yield $r k(\mathbf{D})<4$ which is a contradiction.

- For the second possibility we get $\lambda_{3}=\mu_{3}=\nu_{3}=0$, which means that all 6 platform anchor points are located on a line.

Therefore we can assume that at least one of the five $3 \times 3$ submatrices has maximal rank. W.l.o.g. we can assume that this submatrix corresponds with the equations $U_{2}, U_{3}, U_{4}$. From this system we now compute $f_{1}, f_{2}, f_{3}$ and plug it into $\Lambda_{1}$ and $U_{i}=\left(1+e_{1}^{2}\right) A_{2} F_{i}(i=5,6)$, where $F_{i}$ only depends quadratically on $e_{3}$. For $\mathbf{s}=\mathbf{b} F_{i}$ has 396 terms and for $\mathbf{s}=\mathbf{a} F_{i}$ has 480 additive factors. $\Lambda_{1}$ which is of degree 8 in $e_{1}$ and $e_{3}$ is too large to be computed explicitly, but this is even not necessary for the following argumentation.

In the general case we can compute $e_{3}$ from $F_{5}$ or $F_{6}$. As $e_{3}$ only depends on design parameters it is constant. Therefore the rotational part of this selfmotion span a line in the spherical kinematic image space (cf. [12]), which is the subspace $f_{0}=\ldots=f_{3}=0$ of the Study quadric. It is well known that straight lines of this subspace correspond with rotation about a fixed axis. Therefore the resulting self-motion can only be a Schönflies self-motion.

The above argumentation fails if $F_{5}$ and $F_{6}$ are fulfilled identically. We show that this cannot be the case without yielding a contradiction. We denote the coefficients of $e_{3}^{j}$ of $F_{5}$ by $w_{j}$ and get

$$
w_{1}:=|\mathbf{A}, \mathbf{B}, \mathbf{B a}, \mathbf{t}|_{2}^{5} \quad \text { and } \quad w_{2}-w_{0}:=|\mathbf{A}, \mathbf{B}, \mathbf{A a}, \mathbf{t}|_{2}^{5}
$$

If these two expressions vanish the manipulator is already architecturally singular as Eq. (21) hold. Clearly the same holds for $U_{6}$ by changing the indices.

Now we discuss the case $e_{0}=0$. This implies $e_{1}=0$ or $e_{3}=0$. Both cases yield a Schönflies self-motion, as the rotational parts span a line in the spherical kinematic image space. 


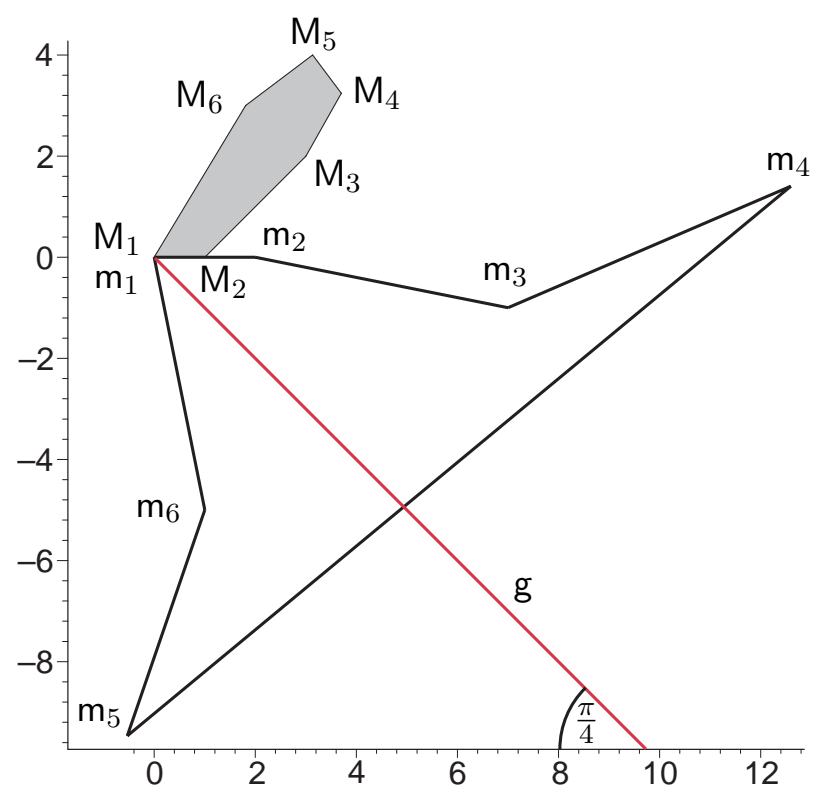

Fig. 1. Geometry of the platform (white) and base (gray).

\section{$7 \quad$ Final example}

The geometry of the non-architecturally singular planar manipulator is given by the coordinates of its anchor points. We have $\mathbf{m}_{1}=\mathbf{M}_{1}=(0,0)^{T}$ and

$$
\begin{aligned}
& \mathbf{m}_{2}=\left(\begin{array}{l}
2 \\
0
\end{array}\right), \quad \mathbf{m}_{3}=\left(\begin{array}{c}
7 \\
-1
\end{array}\right), \mathbf{m}_{4}=\left(\begin{array}{c}
\frac{466}{37} \\
\frac{52}{37}
\end{array}\right), \quad \mathbf{m}_{5}=\left(\begin{array}{c}
\frac{-8}{15} \\
\frac{142}{-15}
\end{array}\right), \mathbf{m}_{6}=\left(\begin{array}{c}
1 \\
-5
\end{array}\right), \\
& \mathbf{M}_{2}=\left(\begin{array}{l}
1 \\
0
\end{array}\right), \quad \mathbf{M}_{3}=\left(\begin{array}{l}
3 \\
2
\end{array}\right), \mathbf{M}_{4}=\left(\begin{array}{c}
\frac{137}{37} \\
\frac{120}{37}
\end{array}\right), \quad \mathbf{M}_{5}=\left(\begin{array}{c}
\frac{47}{15} \\
4
\end{array}\right), \mathbf{M}_{6}=\left(\begin{array}{c}
\frac{29}{16} \\
3
\end{array}\right) .
\end{aligned}
$$

Now we want to inspect if this manipulator is $\mathrm{X}(\mathrm{a})$-singular where a is orthogonal to the base and parallel to the platform. It can easily be seen in Fig. 1 that no 4 anchor points are collinear. Moreover these anchor points fulfill Lemma $1 .{ }^{3}$ Therefore we have to check if there exists an orientation $\sigma$ of the platform such that the 6 conditions $K_{1}=K_{2}=K_{4}=L_{1}=L_{2}=L_{3}=0$ of Theorem 1 are fulfilled for

$$
\left(\begin{array}{l}
A_{i} \\
B_{i}
\end{array}\right):=\mathbf{M}_{i} \quad \text { and } \quad\left(\begin{array}{l}
a_{i} \\
b_{i}
\end{array}\right):=\left(\begin{array}{cc}
\cos \sigma & -\sin \sigma \\
\sin \sigma & \cos \sigma
\end{array}\right) \mathbf{m}_{i}
$$

After applying the half angle substitution $\left(s=\tan \frac{\sigma}{2}\right)$ it can easily be seen that all equations have the factor $s^{2}+2 s-1$ in common which implies the two solutions $\sigma=\pi / 4$ and $\sigma=-3 \pi / 4$, respectively. As a consequence any

$\overline{3}$ If this would not be the case one must relabel the anchor points. 
configuration is singular where the line $\mathrm{g}$ of the moving system is parallel to the base (cf. Fig. 1).

Remark 3 Note that it is not necessary to inspect if the manipulator is also $\mathrm{X}(\mathrm{a})$-singular where a is orthogonal to the platform and parallel to the base, because both properties would already imply an architecturally singular design. This can be seen as follows: If both properties hold then there must exist coordinate systems in the platform and the base with

$$
r k(\mathbf{1}, \mathbf{A}, \mathbf{B}, \mathbf{B b}, \mathbf{a}, \mathbf{b}, \mathbf{A b})_{1}^{6}=r k(\mathbf{1}, \mathbf{A}, \mathbf{B}, \mathbf{B b}, \mathbf{a}, \mathbf{b}, \mathbf{B a})_{1}^{6}=4
$$

(cf. Lemma and Theorem 5). As rk $(\mathbf{1}, \mathbf{A}, \mathbf{B}, \mathbf{a}, \mathbf{b})_{1}^{6}=4$ holds (cf. proof of Theorem 6) Eq. (25) implies $r k(\mathbf{1}, \mathbf{a}, \mathbf{b}, \mathbf{A}, \mathbf{B}, \mathbf{A a}, \mathbf{A b}, \mathbf{B a}, \mathbf{B b})_{1}^{6} \leq 5$. Therefore the manipulator is architecturally singular (cf. $[19,15])$.

\section{Conclusion}

In this paper we presented a complete classification of the set of Schönfliessingular planar parallel manipulators of Stewart Gough type characterized by the property that one of the carrier planes of the platform or base anchor points is orthogonal to the rotational axis a of the Schönflies group X(a). Beside an algebraic characterization (cf. Theorem 1,2,3) also a geometric one (cf. Corrolary 1) was given. Moreover the self-motional behavior of these manipulators was discussed (cf. Theorem 7) and it was shown that this class of planar Stewart Gough platforms possesses a quadratic singularity surface (cf. Theorem 6).

\section{References}

[1] Borel, E.: Mémoire sur les déplacements à trajectoires sphériques, Mém. présentés par divers savants, Paris 2 (33) 1-128 (1908).

[2] Husty, M.L.: An algorithm for solving the direct kinematics of general StewartGough platforms, Mechanism and Machine Theory 31 (4) 365-380 (1996).

[3] Husty, M.L., and Karger, A.: Self motions of Stewart-Gough platforms: an overview, In Proc. of the workshop on fundamental issues and future research directions for parallel mechanisms and manipulators (S.M. Gosselin, I. EbertUphoff eds.), 131-141 (2002).

[4] Karger, A.: Architecture singular planar parallel manipulators, Mechanism and Machine Theory 38 (11) 1149-1164 (2003). 
[5] Karger, A.: Stewart-Gough platforms with simple singularity surface, Advances in Robot Kinematics - Mechanisms and Motion (J. Lenarcic, B. Roth eds.), 247-254, Springer (2006).

[6] Karger, A.: Architecturally singular non-planar parallel manipulators, Mechanism and Machine Theory 43 (3) 335-346 (2008).

[7] Karger, A.: Self-motions of Stewart-Gough platforms, Computer Aided Geometric Design, Special Issue: Classical Techniques for Applied Geometry (B. Jüttler, O. Röschel, E. Zagar, eds.) 25 (9) 775-783 (2008).

[8] Karger, A.: Parallel Manipulators with simple geometrical structure, In Proc. of EuCoMeS'08 (M. Ceccarelli, ed.), 473-480 (2008).

[9] Ma, O., and Angeles, J.: Architecture Singularities of Parallel Manipulators, Int. J. of Robotics and Automation 7 (1) 23-29 (1992).

[10] Merlet, J.-P.: Singular Configurations of Parallel Manipulators and Grassmann Geometry, Int. J. of Robotics Research 8 (5) 45-56 (1992).

[11] Mick, S., and Röschel, O.: Geometry \& architecturally shaky platforms, Advances in Robot Kinematics: Analysis and Control (J. Lenarcic, M.L. Husty eds.), 455-464, Kluwer (1998).

[12] Müller, H.R.: Sphärische Kinematik, VEB Deutscher Verlag der Wissenschaften (1962).

[13] Nawratil, G.: Zur Geometrie von Stewart Gough Plattformen, Master thesis (Supervisor: H. Stachel), Vienna University of Technology (2004).

[14] Nawratil, G.: On the degenerated cases of architecturally singular planar parallel manipulators, J. of Geometry and Graphics 12 (2) 141-149 (2008).

[15] Nawratil, G.: A new approach to the classification of architecturally singular parallel manipulators, Computational Kinematics (A. Kecskemethy, A. Müller eds.), 349-358, Springer (2008).

[16] Nawratil, G.: Main theorem on Schönflies-singular planar Stewart Gough platforms, Advances in Robot Kinematics (J. Lenarcic, M.M. Stanisic eds.), Springer (2010), to appear.

[17] Nawratil, G.: Special cases of Schönflies-singular planar Stewart Gough platforms, New Trends in Mechanisms Science: Mechanisms and Control (D. Pisla et al. eds.), Springer (2010), to appear.

[18] Pottmann, H., and Wallner, J.: Computational Line Geometry. Mathematics + Visualization, Springer (2001).

[19] Röschel, O., and Mick, S.: Characterisation of architecturally shaky platforms, Advances in Robot Kinematics: Analysis and Control (J. Lenarcic, M.L. Husty eds.), 465-474, Kluwer (1998).

[20] Schönflies, A.M.: Über Gruppen von Bewegungen, Mathematische Annalen 28 319-342 (1886) and 29 50-80 (1887). 
[21] Schönflies, A.M.: Über Gruppen von Transformationen des Raumes in sich, Mathematische Annalen 34 172-203 (1889).

[22] Wohlhart, K.: Architectural Shakiness or Architectural Mobility of Platforms, Advances in Robot Kinematics (J. Lenarcic, M.M. Stanisic eds.), 365-374, Kluwer (2000).

[23] Wohlhart, K.: From higher degrees of shakiness to mobility, Mechanism and Machine Theory 45 (3) 467-476 (2010).

\section{Appendix}

In the following the proof of Theorem 4 is given in two parts:

Part [A] W.l.o.g. we can assume that $\mathrm{m}_{1}, \ldots, \mathrm{m}_{5}$ are collinear with $b_{2} \neq 0$ and $a_{i}=a_{2} b_{i} / b_{2}$ for $i=3,4,5$. Then $Q$ splits up into:

$$
|\mathbf{a}, \mathbf{b}|_{(2,6)}\left(2 e_{0} e_{3}\left(x-A_{6} K\right)-\left(e_{0}^{2}-e_{3}^{2}\right)\left(y-B_{6} K\right)\right) F[288] .
$$

1. $\mathrm{m}_{1}, \ldots, \mathrm{m}_{5}$ are pairwise distinct: We consider $F_{101}=b_{2} K U_{1}$ and $F_{100}=$ $b_{2}^{2} K^{2} U_{2}$. Now $U_{1}=0$ and $U_{1}-U_{2}=0$ correspond to the conditions given in Eq. (17) of Karger [6] implying case 10 of the list of architecturally singular manipulators.

2. Assuming $\mathrm{m}_{1}=\mathrm{m}_{4}=\mathrm{m}_{5}$; i.e. $b_{4}=b_{5}=0$. Then $F_{101}$ can only vanish w.c. for $B_{3}=0$. Now $F=z b_{2} b_{3}\left(A_{2}-A_{3}\right)|\mathbf{A}, \mathbf{B}|_{4}^{5}\left(y b_{2} K-2 z a_{2} e_{0} e_{3}\right)$ yields the contradiction.

3. Assuming $\mathrm{m}_{1}=\mathrm{m}_{3}$ : Then $U_{1}=0$ and $F_{010}=0$ imply the collinearity of $M_{2}, M_{4}, M_{5}$. Now the equation $U_{2}=0$ remains, which corresponds to the special case of item 10 of Karger's list of architecturally singular manipulators (cf. [6]).

Part [B] W.l.o.g. we can assume that $\mathrm{m}_{1}, \ldots, \mathrm{m}_{4}$ are collinear with $b_{2} \neq 0$ and $a_{i}=a_{2} b_{i} / b_{2}$ for $i=3,4$. Then $Q$ splits up into $K b_{2}^{2} F[6312]$. In the first step we compute the resultant of $F_{102}^{31}$ and $F_{201}^{31}$ with respect to $A_{2}$ which yields:

$$
b_{2} c_{(1,2,5)} c_{(1,2,6)} B_{3} B_{4}\left(b_{3}-b_{4}\right)\left(B_{5}-B_{6}\right) G[20] .
$$

$B_{3}=0$ :

$\overline{\text { Now }}_{101}^{60}=0$ yields $B_{4} B_{5} B_{6}|\mathbf{A}, \mathbf{b}|_{2}^{3}\left[a_{2} b_{4}\left(b_{5}-b_{6}\right)-a_{5} b_{2}\left(b_{4}-b_{6}\right)+a_{6} b_{2}\left(b_{4}-b_{5}\right)\right]$.

1. $A_{3}=A_{2} b_{3} / b_{2}$ : The resultant of $F_{200}^{51}$ and $F_{101}^{51}-F_{101}^{15}$ with respect to $a_{6}$ can only vanish w.c. in the following two cases:

a. $b_{4}=b_{6}$ : Then $F_{200}^{51}=0$ implies $b_{4}=b_{5}$. From $F_{101}^{51}-F_{101}^{15}=0$ we get $B_{5}=B_{6}$. Then $F_{102}^{22}=0$ implies an expression for $A_{6} . F_{101}^{42}=0$ yields the contradiction. 
b. $B_{5}=B_{6}, b_{4} \neq b_{6}$ : W.l.o.g. we can compute $a_{5}$ from $F_{200}^{51}=0$. Now $F_{021}^{40}=$ 0 implies an expression for $A_{5}$. Again $F_{101}^{42}=0$ yields the contradiction.

2. $\left[a_{2} b_{4}\left(b_{5}-b_{6}\right)-a_{5} b_{2}\left(b_{4}-b_{6}\right)+a_{6} b_{2}\left(b_{4}-b_{5}\right)\right]=0,|\mathbf{A}, \mathbf{b}|_{2}^{3} \neq 0$ :

a. $b_{4} \neq b_{5}$ : Under this assumption we can compute $a_{6}$ from the condition. Then $F_{201}^{31}=0$ and $F_{101}^{51}+F_{101}^{15}=0$ imply $\mathrm{M}_{5}=\mathrm{M}_{6}$. Finally $F_{021}^{40}=0$ yields the contradiction.

b. $b_{4}=b_{5}$ : The condition implies $b_{4}=b_{5}=b_{6}$. From $F_{102}^{31}=0$ we get $B_{5}=B_{6}$.

i. $A_{2} b_{3}\left(b_{6}-b_{2}\right)-A_{3} b_{2}\left(b_{6}-b_{3}\right) \neq 0$ : W.l.o.g. we can compute $A_{6}$ from $F_{021}^{40}=0$. Then $F_{200}^{51}=0$ yields the contradiction.

ii. $A_{2} b_{3}\left(b_{6}-b_{2}\right)-A_{3} b_{2}\left(b_{6}-b_{3}\right)=0$ : W.l.o.g. we can solve this equation for $b_{6}$. Again the conditions $F_{021}^{40}=0$ and $F_{200}^{51}=0$ cannot vanish w.c..

As a consequence of this study we can assume for the remaining discussion that no 3 points of $\mathrm{M}_{1}, \ldots, \mathrm{M}_{4}$ are collinear.

$b_{3}=b_{4}, B_{3} B_{4} \neq 0$ :

In this case $F_{200}^{51}$ yields $b_{4} C_{(2,3,4)} T[8]$. For $b_{4}=0$ the condition $F_{101}^{60}=0$ implies $B_{5}=0$ or $B_{6}=0$. In both cases $F_{102}^{31}=0$ and $F_{201}^{31}=0$ yield the contradiction. Therefore we can assume $b_{3}=b_{4} \neq 0$ and set $T$ equal to zero.

1. $b_{4} \neq b_{6}$ : Under this assumption we can compute $B_{6}$ from $T=0$. Then we compute $F_{101}^{51}+F_{101}^{15}$ which splits up into $B_{5}\left(A_{5}-A_{6}\right)\left(b_{4}-b_{5}\right) c_{(1,2,6)} U[4]$ with $U:=b_{2}\left(A_{3} B_{4}-A_{4} B_{3}\right)+b_{4} A_{2}\left(B_{3}-B_{4}\right)$. As $B_{5}=0$ yields $B_{6}=0$ a contradiction, we have to distinguish the following 3 cases:

a. W.l.o.g. we can solve $U=0$ for $A_{4}$. Then $F_{101}^{51}-F_{101}^{15}=0$ implies an expression for $a_{5}$. From $F_{020}^{60}=0$ we get $A_{5}$. Finally $F_{101}^{42}=0$ yields the contradiction.

b. $b_{4}=b_{5}, U \neq 0$ : Now $F_{102}^{31}=B_{5} U c_{(1,2,5)} c_{(1,2,6)}$ yields a contradiction.

c. $A_{5}=A_{6},\left(b_{4}-b_{5}\right) U \neq 0$ : Now $F_{102}^{31}=0$ implies an expression for $a_{5}$. Then $F_{020}^{60}=0$ yields the contradiction.

2. $b_{4}=b_{6}$ : Then $T$ splits up into $B_{5}\left(b_{5}-b_{6}\right) c_{(1,2,6)}$.

a. $b_{5}=b_{6}$ : In this case $F_{102}^{31}$ factors into $c_{(1,2,5)} c_{(1,2,6)}\left(B_{5}-B_{6}\right) U$.

i. $U=0$ : W.l.o.g. we compute $A_{4}$ from this condition. Then $F_{101}^{51}-$ $F_{101}^{15}=0$ implies $B_{5}=B_{6}$. From $F_{200}^{51}=0$ we get an expression for $A_{6}$. Finally $F_{101}^{42}=0$ yields the contradiction.

ii. $B_{5}=B_{6}, U \neq 0$ : Assuming $B_{3} \neq B_{4}$ we can compute $A_{6}$ from $F_{021}^{40}=0$. Then $F_{200}^{51}=0$ already yields the contradiction. For $B_{3}=$ $B_{4}$ the condition $F_{021}^{40}=0$ implies $a_{5}=a_{6}$. Then $F_{200}^{51}=0$ yields the contradiction.

b. $B_{5}=0, b_{5} \neq b_{6}$ : Now $F_{102}^{31}$ can only vanish w.c. for $U=0$. From this condition we again express $A_{4}$. Finally $F_{101}^{51}-F_{101}^{15}=0$ yields the contradiction. 
$B_{5}=B_{6},\left(b_{3}-b_{4}\right) B_{3} B_{4} \neq 0$ :

$\overline{\text { W.l.o.g. we can express } A_{6}}$ from $F_{201}^{31}=0$.

1. $b_{5} \neq b_{6}$ : Under this assumption we can compute $A_{5}$ from $F_{200}^{51}=0$. Now $F_{100}^{62}$ can only vanish w.c. for $b_{3} b_{4}=0$. In both cases $F_{101}^{42}=0$ implies the contradiction.

2. $b_{5}=b_{6}$ : Here we distinguish the following subcases:

a. $b_{3} \neq b_{6}, b_{4} \neq b_{6}$ : W.l.o.g. we compute $A_{4}$ from $F_{200}^{51}=0$. Now the resultant of $F_{100}^{62}$ and $F_{102}^{22}$ with respect to $B_{6}$ can only vanish w.c. in the following cases:

i. $b_{3} b_{4}=0$ : W.l.o.g. we set $b_{3}=0$. Then $F_{102}^{22}=0$ implies $b_{6}=$ $b_{2}+B_{6}\left(b_{4}-b_{2}\right) / B_{4}$. Finally $F_{101}^{42}=0$ yields the contradiction.

ii. $b_{5}=b_{6}=0, b_{3} b_{4} \neq 0$ : Now $F_{102}^{22}=0$ implies $b_{3}=b_{2}+B_{3}\left(b_{4}-b_{2}\right) / B_{4}$. Again $F_{101}^{42}=0$ yields the contradiction.

iii. $b_{2}=b_{6}, b_{3} b_{4} b_{5} b_{6} \neq 0: F_{100}^{62}=0$ implies the contradiction.

iv. $b_{3}=b_{2}+B_{3}\left(b_{4}-b_{2}\right) / B_{4},\left(b_{2}-b_{6}\right) b_{3} b_{4} b_{5} b_{6} \neq 0$ : Then $F_{100}^{62}=0$ implies $b_{6}=b_{2}+B_{6}\left(b_{4}-b_{2}\right) / B_{4}$. Finally $F_{101}^{42}=0$ yields the contradiction.

b. W.l.o.g. we set $b_{4}=b_{6}$ : In this case $F_{200}^{51}$ can only vanish w.c. for $B_{4}=B_{6}$. Finally $F_{101}^{51}+F_{101}^{15}=0$ yields the contradiction.

$\underline{G[20]=0,\left(B_{5}-B_{6}\right)\left(b_{3}-b_{4}\right) B_{3} B_{4} \neq 0:}$

1. $|\mathbf{B}, \mathbf{b}|_{3}^{4} \neq 0$ : Under this assumption we can express $A_{6}$ from $G=0$. Then the resultant of $F_{102}^{31}$ and $F_{201}^{31}$ with respect to $a_{6}$ can only vanish w.c. for:

a. $|\mathbf{A}, \mathbf{B}, \mathbf{b}|_{2}^{4}=0$ : We compute $A_{2}$ from this condition. Then $F_{101}^{60}=0$ implies an expression for $A_{5}$. Finally $F_{002}^{60}=0$ yields the contradiction.

b. $|\mathbf{B}, \mathbf{b}, \mathbf{B b}|_{(3,4,6)}=0,|\mathbf{A}, \mathbf{B}, \mathbf{b}|_{2}^{4} \neq 0$ :

i. $B_{4} b_{3}\left(b_{4}-b_{6}\right)-B_{3} b_{4}\left(b_{3}-b_{6}\right) \neq 0$ : Under this assumption we can compute $B_{6}$ from $|\mathbf{B}, \mathbf{b}, \mathbf{B b}|_{(3,4,6)}=0$.

$\star B_{4} b_{3}\left(b_{4}-b_{5}\right)-B_{3} b_{4}\left(b_{3}-b_{5}\right) \neq 0$ : Now we can compute $B_{5}$ from $F_{201}^{31}=0$. Finally $F_{102}^{31}=0$ yields the contradiction.

$\star B_{4} b_{3}\left(b_{4}-b_{5}\right)-B_{3} b_{4}\left(b_{3}-b_{5}\right)=0$ : W.l.o.g. we can express $b_{5}$ from this condition. Now $F_{201}^{31}$ can only vanish w.c. for $b_{3}=0, b_{4}=0$ or $B_{3}=B_{4}$. In all three cases $F_{102}^{31}=0$ yields the contradiction.

ii. $B_{4} b_{3}\left(b_{4}-b_{6}\right)-B_{3} b_{4}\left(b_{3}-b_{6}\right)=0$ : W.l.o.g. we can express $b_{6}$ from this condition. Then $|\mathbf{B}, \mathbf{b}, \mathbf{B b}|_{(3,4,6)}=0$ can only vanish w.c. for $b_{3}=0, b_{4}=0$ or $B_{3}=B_{4}$. In all three cases the conditions $F_{102}^{31}=0$ and $F_{201}^{31}=0$ yield the contradiction.

2. $|\mathbf{B}, \mathbf{b}|_{3}^{4}=0$ : We solve this condition for $b_{3}$. Now $G$ splits up into $C_{(1,3,4)} N[6]$. W.l.o.g. we can compute $B_{6}$ from $N=0$. Then we can express $B_{5}$ from $F_{102}^{31}=0$. Finally $F_{201}^{31}=0$ implies the contradiction. End of all cases. 